\title{
An Efficient Parameter Calibration Method for API Hydrological Model
}

\author{
Jiang $\mathrm{Wu}^{1, \text { a }}$, Jianzhong Zhou ${ }^{2, \mathrm{~b}}$, Shuang Zhu ${ }^{1, \mathrm{c}}$, and Hairong Zhang ${ }^{1, \mathrm{~d}}$, \\ Jun Guo $3,4, \mathrm{e}$ \\ ${ }^{1}$ School of Hydropower and Information Engineering, Huazhong University of Science and \\ Technology, Wuhan 430074, China \\ 2 Hubei Key Laboratory of Digital Valley Science and Technology, Wuhan 430074, China \\ ${ }^{3}$ State Key Laboratory of Disaster Prevention and Reduction for Power Grid Transmission and \\ Distribution Equipment \\ ${ }^{4}$ State Grid Hunan Electric Power Company Disaster prevention and reduction center \\ a hae_wujiang@hust.edu.cn, bjz.zhou@hust.edu.cn, ${ }^{c}$ zhushuang@hust.edu.cn, ${ }^{\mathrm{d}}$ zhr@hust.edu.cn \\ e332413817@qq.com
}

\begin{abstract}
Keywords: API hydrological model; parameter calibration method; rainfall-runoff correlation diagram; instantaneous unit hydrograph

Abstract: API hydrological model is common used on streamflow forecasting for its' simple structure and robust ability on acquiring high precision forecasting results. But for a long time, parameter calibration has been the major difficulty for the use of API hydrological model. API model develops from the empirical approach. It's more difficult than other hydrological models to achieve the aim of fully automatic parameter calibration without artificial experience processing. In this paper, a method is proposed for enhancing the efficiency of parameter calibration for API hydrological model. In this method, the rainfall-runoff correlation diagram is drawn according to rainfall and flood data and instantaneous unit hydrograph is adopted to calculate the outflow by net rainfall. What's more, an intelligent algorithm is employed for the solution of parameters of API hydrological model. The result shows that the proposed method is efficient on parameter calibration for API hydrological model.
\end{abstract}

\section{Introduction}

API (Antecedent Precipitation Index) model is a traditional hydrological forecasting model, the main contents of which are rainfall-runoff correlation diagram and unit hydrograph. The basis of rainfall-runoff correlation forecasting method is built on the early manual rainfall-runoff forecasting method. The first step is to calculate antecedent influence rainfall $(\mathrm{Pa})$, which means antecedent soil moisture. After that, the average precipitation and runoff of each flood is computed to get discrete point set which is used to fit the rainfall-runoff correlation diagram. The unit hydrograph is the process of surface runoff at the export section formed by a unit of net rainfall on the river basin. For its' simple structure and robust ability on acquiring high precision forecasting results, API model is common used on streamflow forecasting [1 3]. Shakir Ali [4] proposed normalized antecedent precipitation index (NAPI) model and it was applied to three watersheds in the semi-arid region of India to simulate runoff yield. The proposed model showed improved correlation between the observed and predicted runoff data compared to the Soil Conservation Service Curve Number (SCS-CN) model. Tuhua Ma [5] developed a methodology accounting for the effective antecedent rainfall from a power-law relationship between the frequency of landslide occurrence and the landslide-triggering rainfall level and the results had significantly practical implications. However, there exist many empirical experience processes in the modeling of API model. Parameter calibration has been the major difficulty for the use of API hydrological model. With the improvement of computing technique, automatic parameter calibration is becoming rigid requirement for hydrological forecasting model. 


\section{Basic concepts}

API model contains runoff yield and concentration process. Rainfall-runoff correlation diagram is drawn according to rainfall and flood data observed in the pastime and used to calculate net rainfall in the runoff yield process. There are serval types of rainfall-runoff correlation curves in which the most popular one is $P \sim P a \sim R$ curve where $P$ denotes the precipitation, $P a$ denotes the antecedent influence rainfall, and $R$ denotes the runoff. $P \sim P a \sim R$ curve is made up of serval lines that characterize the relation of precipitation and runoff under equal value intervals of antecedent influence rainfall. $P a$ is calculated as:

$$
P_{a, t+1}=k\left(P_{a, t}+P_{t}\right) \text {. }
$$

Where $P_{a, t}$ denotes the antecedent influence rainfall at time $t, P_{t}$ denotes the precipitation at time $t$, $k$ denotes the regression coefficient of the soil.

Unit hydrograph is adopted to calculate the outflow of river basin in the concentration process. Time unit hydrograph is a more common option. But it's rather time-consuming to make the time unit hydrograph since rainfall and flood data observed in the pastime are needed and it requires repeated test. Instantaneous unit hydrograph is more convenient to be calculated. By assuming that the operation performed by the catchment on an instantaneous rainfall is equivalent to a succession of routings through linear storage, instantaneous unit hydrograph is calculated as:

$$
u(0, t)=\frac{1}{K \Gamma(n)}\left(\frac{t}{k}\right)^{n-1} e^{-\frac{t}{k}} .
$$

Where $\Gamma$ denotes the gamma function, $t$ denotes the time, $n$ denotes the parameter that reflect the regulation capacity of watershed, $K$ denotes the storage and discharge coefficient of linear storage.

\section{Parameter Calibration Method}

Multiple floods occurred in the pastime on the river basin are selected as the research object. The precipitation, evaporation and outflow data observed during the flood period will be utilized for parameter calibration. Rainfall-runoff correlation diagram is drawn according to multiple floods data and saved as discrete points in an array. The value of runoff is calculated by precipitation and antecedent influence rainfall using the interpolation method. The parameters $n$ and $K$ of the instantaneous unit hydrograph are selected as decision variables. The flood peak flow and flood volume are the main elements that is concerned in the flood forecasting. So we choose the sum of qualified rate of flood peak flow and average determinacy coefficient (DC) as the objective function. The problem is described as below:

$$
\operatorname{Max} F(n, K)=\operatorname{Max}\left(Q R_{f p}+\frac{1}{m} \sum_{i=1}^{m} D C_{i}\right) .
$$

Where $Q R_{f p}$ denotes the qualified rate of flood peak flow, $m$ denotes the number of floods, DC denotes the determinacy coefficient of each flood which is calculated as:

$$
\left.D C=1-\left[\sum_{i=1}^{n}\left(Q_{i}-\hat{Q}_{\mathrm{i}}\right)^{2}\right] / \sum_{i=1}^{n}\left(Q_{i}-\bar{Q}_{i}\right)^{2}\right] .
$$

Differential Evolution (DE) algorithm is adopted to solve the problem. Differential Evolution (DE) algorithm is firstly proposed by Storn and Price [6]. One form of DE algorithm is the DE/rand/1/bin strategy which contains mutation, crossover and selection operators. This form of DE algorithm is adopted to solve the problem above. 


\section{Case Study}

Zhexi reservoir is located on Zishui river basin in the middle of China's Hunan province. The catchment area above Zhexi reservoir is 22640 square kilometers, accounting for eighty percent of the whole river basin. The mean annual precipitation of Zishui river basin is 1400 millimeter and the precipitation is the only source of runoff in the Zishui river basin. The precipitation mainly concentrates on April, May, and June. The duration of heavy rainfall is usually 1 4 days, and up to six days. There are three forms of floods occurring in the river basin which consists of large quantity $\&$ high peak flood, little quantity \& high peak flood, and the combination of two kinds of floods above. The formation of large quantity \& high peak flood is caused by the rainfall on the whole river basin or on the upper basin. While the little quantity \& high peak flood is caused by the heavy rainfall on the lower basin near to the reservoir. The hourly precipitation and runoff data from year of 2004 to 2014 are adopted for the parameters calibration process.

The floods during year of 2004 to 2014 are chosen for the parameters calibration. The $\mathrm{P} \sim \mathrm{Pa} \sim \mathrm{R}$ curve is shown as Figure 1. Floods from year of 2004 to 2011 are set for parameters calibration, and floods from year of 2012 to 2014 are set for parameters validation. By applying DE algorithm to solve the problem above, the parameters of instantaneous unit hydrograph is calculates as: $\mathrm{n}=3.36, \mathrm{~K}=2.88$. The instantaneous unit hydrograph is transferred to unit hydrograph by $\mathrm{S}$ curve and it is shown as Figure 2. The accuracy estimation results of flood forecasting in calibration period and validation period are shown in Table 1.

From Table 1, the results shows that the average determinacy coefficient is 0.8 and the qualified rate of flood peak flow is $82.05 \%$ in the calibration period. The average determinacy coefficient is 0.827 and the qualified rate of flood peak flow is $80 \%$ in the validation period. In the calibration period, nearly $70 \%$ of floods are simulated well and the determinacy coefficient of these floods are larger than 0.8 . There are also a few floods that are simulated badly such as NO. 15, 16, 20, 21 flood. We found that the relationship of rainfall and runoff are not so matching. The mistake of observing may have something to do with it. However, the results show that the grade of accuracy of the forecasting result has reached grade B which can be used for floods forecasting. What's more, this method has greatly enhances the efficiency of parameter calibration for API hydrological model.

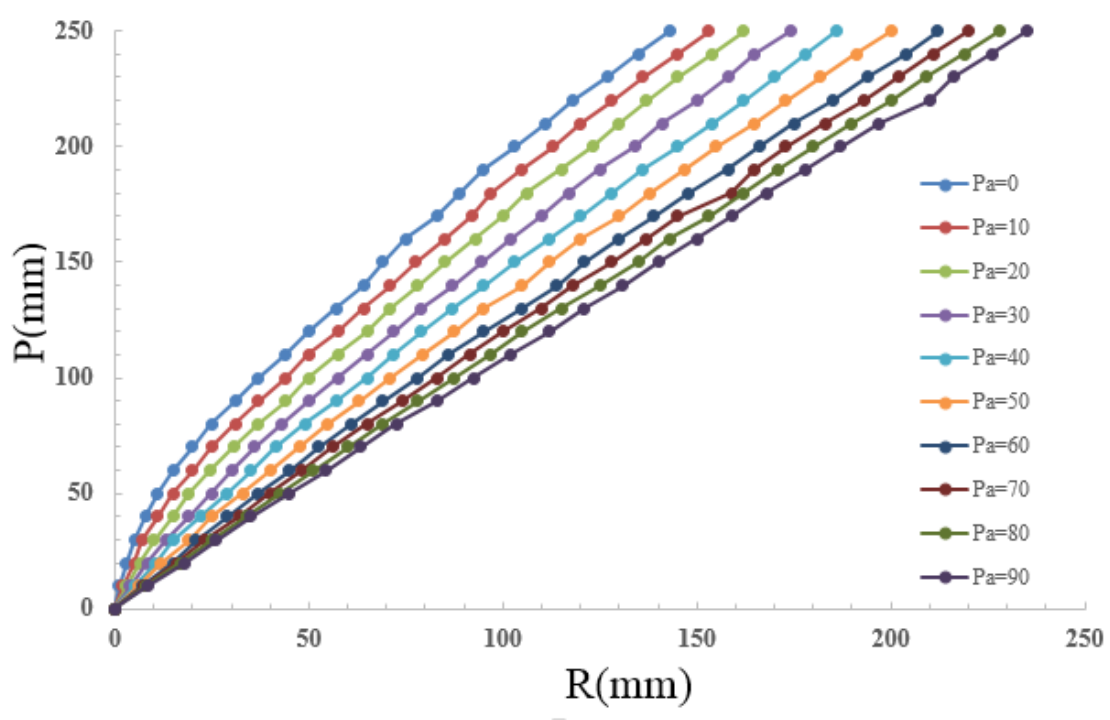

Figure 1 P Pa $\sim R$ curve 


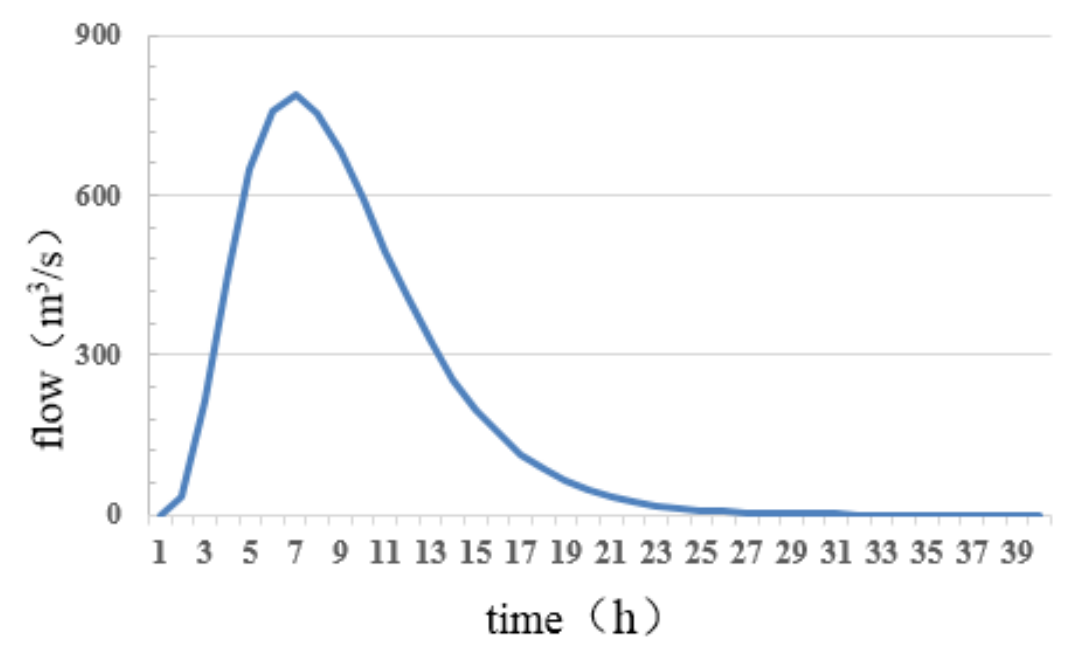

Figure 2 Unit hydrograph

Table 1 The accuracy estimation results of flood forecasting

\begin{tabular}{|c|c|c|c|c|c|}
\hline Period & $\begin{array}{c}\text { Flood } \\
\text { numb } \\
\text { er }\end{array}$ & DC & $\begin{array}{c}\text { Observed flood } \\
\text { peak }\left(\mathbf{m}^{3} / \mathbf{s}\right)\end{array}$ & $\begin{array}{c}\text { Forecast flood } \\
\text { peak }\left(\mathbf{m}^{3} / \mathbf{s}\right)\end{array}$ & $\begin{array}{c}\text { Relative error } \\
\text { of flood peak }\end{array}$ \\
\hline \multirow{31}{*}{$\begin{array}{l}\text { Calibration } \\
\text { Period }\end{array}$} & 1 & 0.58 & 2009.7 & 2298.92 & 0.14 \\
\hline & 2 & 0.93 & 5232.78 & 4587.71 & 0.12 \\
\hline & 3 & 0.93 & 3972.79 & 3396.95 & 0.14 \\
\hline & 4 & 0.88 & 6927.92 & 4953.11 & 0.29 \\
\hline & 5 & 0.94 & 4735.06 & 3502.92 & 0.26 \\
\hline & 6 & 0.91 & 2545.88 & 2379.69 & 0.07 \\
\hline & 7 & 0.96 & 6100.96 & 5813.47 & 0.05 \\
\hline & 8 & 0.90 & 5483.7 & 4664.03 & 0.15 \\
\hline & 9 & 0.87 & 4948.37 & 4572.65 & 0.08 \\
\hline & 10 & 0.78 & 2141 & 2189.74 & 0.02 \\
\hline & 11 & 0.88 & 2995.15 & 3324.19 & 0.11 \\
\hline & 12 & 0.93 & 6364.27 & 5935.58 & 0.07 \\
\hline & 13 & 0.84 & 3480.3 & 3280.70 & 0.06 \\
\hline & 14 & 0.81 & 3480.3 & 3280.70 & 0.06 \\
\hline & 15 & 0.45 & 2089.32 & 2224.82 & 0.06 \\
\hline & 16 & 0.23 & 2011.07 & 2632.33 & 0.31 \\
\hline & 17 & 0.80 & 6222.45 & 6372.26 & 0.02 \\
\hline & 18 & 0.86 & 2078.61 & 2104.72 & 0.01 \\
\hline & 19 & 0.83 & 4593.68 & 4351.48 & 0.05 \\
\hline & 20 & 0.50 & 2806.35 & 3553.46 & 0.27 \\
\hline & 21 & 0.30 & 2047.05 & 2637.13 & 0.29 \\
\hline & 22 & 0.72 & 3080.32 & 2181.27 & 0.29 \\
\hline & 23 & 0.77 & 4619.13 & 4711.72 & 0.02 \\
\hline & 24 & 0.80 & 3278.95 & 3514.47 & 0.07 \\
\hline & 25 & 0.96 & 7206.82 & 6788.12 & 0.06 \\
\hline & 26 & 0.82 & 2850.92 & 3426.32 & 0.20 \\
\hline & 27 & 0.90 & 6678.16 & 6783.87 & 0.02 \\
\hline & 28 & 0.87 & 7331.16 & 7454.37 & 0.02 \\
\hline & 29 & 0.92 & 9173.75 & 8262.13 & 0.10 \\
\hline & 30 & 0.78 & 1783.5 & 1715.14 & 0.04 \\
\hline & 31 & 0.79 & 3394.46 & 2778.93 & 0.18 \\
\hline
\end{tabular}




\begin{tabular}{|c|c|c|c|c|c|}
\hline \multirow{5}{*}{} & 32 & 0.89 & 4935.15 & 4433.33 & 0.10 \\
\cline { 2 - 6 } & 33 & 0.92 & 7264.33 & 6779.44 & 0.07 \\
\cline { 2 - 6 } & 34 & 0.77 & 2026.88 & 2447.91 & 0.21 \\
\cline { 2 - 6 } & 35 & 0.87 & 5360.06 & 5854.89 & 0.09 \\
\cline { 2 - 6 } & 36 & 0.81 & 3326.6 & 2801.86 & 0.16 \\
\cline { 2 - 6 } & 37 & 0.89 & 2779.37 & 2458.61 & 0.12 \\
\cline { 2 - 6 } period & 38 & 0.83 & 4013.71 & 4030.57 & 0.00 \\
\cline { 2 - 6 } & 49 & 0.69 & 2736.46 & 3294.15 & 0.20 \\
\cline { 2 - 6 } & 40 & 0.93 & 6667.75 & 7636.62 & 0.15 \\
\cline { 2 - 6 } & 42 & 0.87 & 2567.95 & 2335.59 & 0.09 \\
\cline { 2 - 6 } & 43 & 0.64 & 3501.31 & 3764.83 & 0.08 \\
\cline { 2 - 6 } & 44 & 0.78 & 3409.64 & 4234.48 & 0.24 \\
\cline { 2 - 6 } & 45 & 0.64 & 5236.95 & 4964.78 & 0.05 \\
\cline { 2 - 6 } & 46 & 0.78 & 3743.8 & 4047.00 & 0.08 \\
\cline { 2 - 6 } & 47 & 0.91 & 7447.9 & 6295.09 & 0.15 \\
\cline { 2 - 6 } & 48 & 0.82 & 4061.52 & 2858.14 & 0.30 \\
\cline { 2 - 6 } & 49 & 0.96 & 9114.51 & 7660.35 & 0.16 \\
\hline
\end{tabular}

\section{Conclusions}

In this paper, we have proposed a method for enhancing the efficiency of parameter calibration for API hydrological model. By applying this method on the Zhexi reservoir, we had drawn the rainfall-runoff correlation diagram based on the history data of precipitation and runoff and obtained the parameters of instantaneous unit hydrograph by the utilization of DE algorithm to solve the optimization problem. The results show that the grade of accuracy of the forecasting result has reached grade B which can be used for floods forecasting. Moreover, the proposed method has greatly enhances the efficiency of parameter calibration for API hydrological model. So, we consider that this method is efficient on parameter calibration for API hydrological model.

\section{Acknowledgements}

This work is supported by the National Natural Science Foundation of China (NSFC) (No.51239004, No. 91547208 and No. 51579107).

\section{References}

[1] Fedora M, Beschta R: Journal of Hydrology 112:121-133. (1989)

[2] Heggen RJ: Journal of Hydrologic Engineering 6:377-381. (2001)

[3] Descroix L, Nouvelot J-F, Vauclin M: Journal of Hydrology 263:114-130. (2002)

[4] Ali S, Ghosh N, Singh R: Hydrological Sciences Journal-Journal des Sciences Hydrologiques 55:266-274. (2010)

[5] Ma T, Li C, Lu Z, Wang B: Geomorphology 216:187-192. (2014)

[6] Storn R, Price K: International Computer Science Institute, Berkeley Berkeley, CA. (1995) 